\title{
Exact Values of Bernstein $n$-Widths for Some Classes of Convolution Functions
}

\author{
Feng Guo ${ }^{1,2}$ \\ ${ }^{1}$ School of Mathematical Sciences, Beijing Normal University, Beijing 100875, China \\ 2 Department of Mathematics, Taizhou University, Taizhou, Zhejiang 317000, China
}

Correspondence should be addressed to Feng Guo, gfeng@tzc.edu.cn

Received 10 December 2007; Revised 7 April 2008; Accepted 16 June 2008

Recommended by Vijay Gupta

We consider some classes of $2 \pi$-periodic convolution functions $\widetilde{B}_{p}$, and $\widetilde{K}_{p}$, which include the classical Sobolev class as a special case. With the help of the spectra of nonlinear integral equations, we determine the exact values of Bernstein $n$-width of the classes $\widetilde{B}_{p}, \widetilde{K}_{p}$ in the space $L^{p}$ for $1<p<\infty$.

Copyright (C) 2008 Feng Guo. This is an open access article distributed under the Creative Commons Attribution License, which permits unrestricted use, distribution, and reproduction in any medium, provided the original work is properly cited.

\section{Introduction and main results}

Let $X$ be a normed linear space and let $A$ be a subset of $X$. Assume that $A$ is closed, convex, and centrally symmetric (i.e., $x \in A$ implies $-x \in A$ ). The Bernstein $n$-width, which was originally introduced by Tikhomirov [1], of $A$ in $X$ is given by

$$
b_{n}(A ; X)=\sup _{X_{n+1}} \sup \left\{\lambda: \lambda S\left(X_{n+1}\right) \subseteq A\right\},
$$

where $S\left(X_{n+1}\right)=\left\{x: x \in X_{n+1},\|x\| \leq 1\right\}$ and $X_{n+1}$ is taken over all subspaces of $X$ of dimension at least $n+1$. Let $\mathbb{T}:=[0,2 \pi)$ be the torus, and as usual, let $L^{q}:=L^{q}[0,2 \pi]$ be the classical Lebesgue integral space of $2 \pi$-periodic real-valued functions with the usual norm $\|\cdot\|_{q}, 1 \leq q \leq$ $\infty$.

Denote by $W_{p}^{r}$ the classical Sobolev class of real functions $f$ whose $(r-1)$ th derivative is absolutely continuous and whose $r$ th derivative satisfies the condition $\left\|f^{(r)}\right\|_{q} \leq 1$. The concept of Bernstein $n$-width for the Sobolev classes $W_{p}^{r}$ was originally introduced by Tikhomirov [1]. He considered $b_{n}\left(W_{p}^{r} ; L^{q}\right), 1 \leq p, q \leq \infty$, and found the exact value of $b_{2 n-1}\left(W_{\infty}^{r} ; L^{\infty}\right)$. Pinkus [2] obtained the exact value of $b_{2 n-1}\left(W_{1}^{r} ; L^{1}\right)$. Later, Magaril-Il'yaev [3] obtained the exact value 
of $b_{2 n-1}\left(W_{p}^{r} ; L^{p}\right), 1<p<\infty$. The latest contribution to this field is due to Buslaev et al. [4] who found the exact values of $b_{2 n-1}\left(W_{p}^{r} ; L^{q}\right)$ for all $1<p \leq q<\infty$.

Definition 1.1 (see [2, page 129]). A real, $2 \pi$-periodic, continuous function $G$ satisfies property $B$ if for every choice of $0 \leq t_{1}<\cdots<t_{m}<2 \pi$ and each $m \in \mathbb{N}$, the subspace

$$
X_{m}:=\left\{b+\sum_{j=1}^{m} b_{j} G\left(\cdot-t_{j}\right): \sum_{j=1}^{m} b_{j}=0\right\}
$$

is of dimension $m$, and is a weak Tchebycheff- (WT-) system (see [2, page 39]) for all $m$ odd. A real, $2 \pi$-periodic, continuous function $G$ is said to be $B$-kernel if $G$ satisfies property $B$.

Definition 1.2 (see $[2$, pages 60,126$]$ ). Assume that $K$ is a real, continuous, $2 \pi$-periodic function. One says that $K$ is a cyclic variation diminishing kernel of order $2 m-1\left(\mathrm{CVD}_{2 m-1}\right)$ if there exist $\sigma_{n} \in\{-1,1\}, n=1, \ldots, m$, such that

$$
\sigma_{n} \operatorname{det}\left(K\left(x_{i}-y_{j}\right)\right)_{i, j=1}^{2 n-1} \geq 0
$$

for all $x_{1}<\cdots<x_{2 n-1}<x_{1}+2 \pi$ and $y_{1}<\cdots<y_{2 n-1}<y_{1}+2 \pi$. One will drop the subscript $2 m-1$ from the acronyms CVD, if one assumes that these properties hold for all orders. One says that $K$ is nondegenerate cyclic variation diminishing (NCVD) if $K$ is nonnegative CVD and

$$
\operatorname{dim} \operatorname{span}\left\{K\left(x_{1}-\cdot\right), \ldots, K\left(x_{n}-\cdot\right)\right\}=n,
$$

for every choice of $0 \leq x_{1}<\cdots<x_{n}<2 \pi$ and all $n \in \mathbb{N}$.

Now, we introduce the classes of functions to be studied. Let $K$ be a NCVD kernel [2] and let $G$ be a $B$-kernel. The $2 \pi$-periodic convolution function classes $\widetilde{K}_{p}$ and $\widetilde{B}_{p}$ are defined as follows:

$$
\begin{gathered}
\widetilde{B}_{p}:=\left\{f: f(x)=(G * h)(x)+a, a \in \mathbb{R}, h \perp 1,\|h\|_{p} \leq 1\right\}, \\
\widetilde{K}_{p}:=\left\{f: f(x)=(K * h)(x), h \perp 1,\|h\|_{p} \leq 1\right\},
\end{gathered}
$$

where

$$
(g * h)(x):=\int_{\mathbb{T}} g(x-y) h(y) d y
$$

and $h \perp 1$ means $\int_{\mathbb{T}} h(y) d y=0$.

The exact values of $b_{n}\left(\widetilde{B}_{p} ; L^{q}\right)$ and $b_{n}\left(\tilde{K}_{p} ; L^{q}\right)$ are known for the cases $p=q=1, p=$ $q=\infty$, and $n$ is odd (see [2] for more details). Chen [5] is the one who found the lower estimate of $b_{2 n-1}\left(\tilde{B}_{p}, L^{p}\right)$ and $b_{2 n-1}\left(\tilde{K}_{p}, L^{p}\right)$ for $1<p<\infty$. In this paper, we will determine the exact constants of some classes of periodic convolution functions $\widetilde{B}_{p}$ with $B$-kernel (or NCVDkernel) for $p \in(1, \infty)$, which include the classical Sobolev class as its special case. 
Feng Guo

Now, we are in a position to state our main results of this paper.

Theorem 1.3. Let $G$ be a B-kernel, and $n=1,2, \ldots$ Then

$$
\begin{gathered}
b_{2 n-1}\left(\widetilde{B}_{p} ; L^{p}\right)=\lambda_{n}(p, p, G), \quad 1<p<\infty, \\
s_{2 n}\left(\widetilde{B}_{p} ; L^{p}\right)=b_{2 n-1}\left(\widetilde{B}_{p} ; L^{p}\right)=\lambda_{n}(p, p, G),
\end{gathered}
$$

where

$$
\begin{aligned}
& D_{n}:=\left\{h: h\left(x+\frac{\pi}{n}\right)=-h(x), h(x)\{\sin \} n x \geq 0,\|h\|_{p} \leq 1\right\}, \\
& \lambda_{n}:=\lambda_{n}(p, q, G)=\{\sup \}\left\{\|G * h\|_{q}: h \in D_{n},\right\}, \quad 1<q \leq p<\infty,
\end{aligned}
$$

and $s_{n}\left(\widetilde{B}_{p} ; L^{p}\right)$ denotes any one of the three $n$-widths, Kolmogorov, Gel'fand and $[2$, pages $1 ; 7 ; 20]$.

Theorem 1.4. Let $K$ be a $\{\mathrm{NCVD}\}$ kernel and $n=1,2, \ldots$ Then

$$
\begin{gathered}
b_{2 n-1}\left(\tilde{K}_{p} ; L^{p}\right)=\lambda_{n}(p, p, K), \quad 1<p<\infty, \\
s_{2 n}\left(\tilde{K}_{p} ; L^{p}\right)=b_{2 n-1}\left(\tilde{K}_{p} ; L^{p}\right)=\lambda_{n}(p, p, K), \\
\lambda_{n}(p, q, K)=\{\sup \}\left\{\|K * h\|_{q}: h \in D_{n},\right\}, \quad 1<q \leq p<\infty .
\end{gathered}
$$

We will only give the proof for the case of a B-kernel. As for the case of a NCVD kernel, the proof is similar and even more simple.

\section{Nonlinear integral equation and its spectral couple}

Before we prove Theorem 1.3, we need some results about nonlinear integral equations and their spectral couple. First, we introduce some definitions and notations.

Definition 2.1 (see [2, pages 45,59$])$. Let $x=\left(x_{1}, \ldots, x_{n}\right) \in \mathbb{R}^{n} \backslash\{0\}$ be a real nontrivial vector.

(i) $S^{-}(x)$ indicates the number of sign changes in the sequence $x_{1}, \ldots, x_{n}$ with zero terms discarded. The number $S_{c}^{-}(x)$ of cyclic variations of sign of $x$ is given by

$$
S_{c}^{-}(x):=\max _{i} S^{-}\left(x_{i}, x_{i+1}, \ldots, x_{n}, x_{1}, \ldots, x_{i}\right)=S^{-}\left(x_{k}, \ldots, x_{n}, x_{1}, \ldots, x_{k}\right)
$$

where $k$ is some integer for which $x_{k} \neq 0$. Obviously, $S_{c}^{-}(x)$ is invariant under cyclic permutations, and $S_{c}^{-}(x)$ is always an even number.

(ii) $S^{+}(x)$ counts the maximum number of sign changes in the sequence $x_{1}, \ldots, x_{n}$ where zero terms are arbitrarily assigned values +1 or -1 . The number $S_{c}^{+}(x)$ of maximum cyclic variations of sign of $x$ is defined by

$$
S_{c}^{+}(x):=\max _{i} S^{+}\left(x_{i}, x_{i+1}, \ldots, x_{n}, x_{1}, \ldots, x_{i}\right)
$$


Let $f$ be a piecewise continuous, $2 \pi$-periodic, real-valued function on $\mathbb{R}$. One assumes that $f(x)=[f(x+)+f(x-)] / 2$ for all $x$ and

$$
S_{c}(f):=\sup S_{c}^{-}\left(\left(f\left(x_{1}\right), \ldots, f\left(x_{m}\right)\right)\right),
$$

where the supremum is taken over all $x_{1}<\cdots<x_{m}<x_{1}+2 \pi$ and all $m \in \mathbb{N}$.

Moreover, one needs further counts of zeros of a function. Suppose that $f$ is a continuous, $2 \pi$-periodic, real-valued function on $\mathbb{R}$. One defines

$$
\tilde{Z}_{c}(f):=\sup S_{c}^{+}\left(\left(f\left(x_{1}\right), \ldots, f\left(x_{m}\right)\right)\right),
$$

where the supremum runs over all $x_{1}<\cdots<x_{m}<x_{1}+2 \pi$ and all $m \in \mathbb{N}$. Assume that $f$ is a $2 \pi$-periodic, real-valued function on $\mathbb{R}$ for which $f$ is sufficiently smooth. The number of zeros of $f$ on a period, counting multiplicities, is denoted by $Z_{c}^{\star}(f)$.

Clearly, $S_{c}(f)$ denotes the number of sign changes of $f$ on a period, and $\widetilde{Z}_{c}(f)$ denotes the number of zeros of $f$ on a period, where the zeros which are sign changes are counted once and zeros which are not sign changes are counted twice. Moreover, we have

$$
S_{c}(f) \leq \tilde{Z}_{c}(f) \leq Z_{c}^{\star}(f)
$$

We define $Q_{p}$ to be the nonlinear transformation:

$$
\left(Q_{p} f\right)(t):=|f(t)|^{p-1} \operatorname{sign} f(t), \quad 1<p<\infty .
$$

Since the function $F(y):=|y|^{p-1}$ sign $y$ is continuous and strictly increasing, $Q_{p} f$ is continuous if and only if $f$ is. Moreover, since $F(y)$ is uniformly continuous on every compact interval, $Q_{p} f$ is a continuous operator from $C(\mathbb{T})$ to $C(\mathbb{T})$. It is clear that if $f \in L^{p}, 1<p<\infty$, then $Q_{p} f \in L^{p^{\prime}}, p^{\prime}=p /(p-1)$, and $Q_{p^{\prime}} Q_{p} f=f$ for every $f$. For $1 \leq q, p<\infty,\left(f, \lambda^{q}\right)$ is called a spectral couple, and $f$ is called a spectral function if

$$
\begin{aligned}
\|h\|_{p} & =1, \quad f(x)=(G * h)(x)+\beta, \\
\left(Q_{p} h\right)(y) & =\lambda^{-q} \int_{\mathbb{T}} G(x-y)\left(Q_{q} f\right)(x) d x,
\end{aligned}
$$

where $\beta$ satisfies the condition

$$
\inf _{c \in \mathbb{R}}\|(G * h)+c\|_{q}=\|(G * h)+\beta\|_{q}
$$

when $\int_{\mathbb{T}} G(x) d x=0$. It is well known that if $1<q<\infty$, then $\beta$ is unique. The set of all spectral couples is denoted by $\Gamma(p, q, G)$, and the spectral class $\Gamma_{2 n}(p, q, G)$ is given by

$$
\Gamma_{2 n}(p, q, G):=\left\{\left(f, \lambda^{q}\right) \in \Gamma(p, q, G): S_{c}(f)=2 n\right\} .
$$

Lemma 2.2 (see [2, page 177]). Let $\phi$ be a real piecewise continuous $2 \pi$-periodic function satisfying $\phi \perp \mathbf{1}$ and set $\psi(x):=a+(G * \phi)(x)$. If $G$ satisfies property $B$, then

$$
\tilde{Z}_{c}(\psi) \leq S_{c}(\phi)
$$


Lemma 2.3. For $1<p, q<\infty$, if $\left(f, \lambda^{q}\right) \in \Gamma(p, q, G)$ with $S_{c}(h)<\infty$. Then, $f$ has a finite number of zeros, and all its zeros are simple.

Proof. By (2.7) and Lemma 2.2, we have $S_{c}(f) \leq \tilde{Z}_{c}(f) \leq S_{c}(h) \leq \tilde{Z}_{c}\left(Q_{p} h\right) \leq S_{c}\left(Q_{q} f\right)=S_{c}(f)$. Obviously, $S_{c}(f)=S_{c}(h)=\widetilde{Z}_{c}(f)$. Therefore, $f$ has a finite number of zeros, and all its zeros are simple.

Lemma 2.4. (a) If $1<q<p<\infty$, and $f_{1}$ and $f_{2}$ are two spectral functions, then

$$
S_{c}\left(f_{1}+f_{2}\right) \leq \max \left\{S_{c}\left(f_{1}\right), S_{c}\left(f_{2}\right)\right\}<\infty .
$$

(b) If $1<q \leq p<\infty$, and $f_{1}$ and $f_{2}$ correspond to the same spectral value and $f_{1} \neq f_{2}$, then all the zeros of $f_{1}+f_{2}$ are with sign changes.

Proof. Suppose that $\left(f_{1}, \lambda_{1}^{q}\right)$ and $\left(f_{2}, \lambda_{2}^{q}\right)$ are spectral couples and, say $0<\lambda_{1} \leq \lambda_{2}$. For $\varepsilon>0$, let $\sigma(\varepsilon):=S_{c}\left(f_{1}+\varepsilon f_{2}\right)$. For all sufficiently small $\varepsilon$, we have $\sigma(\varepsilon)=S_{c}\left(f_{1}\right)=\tilde{Z}_{c}\left(f_{1}\right)=: N$. Indeed, let $t_{1}, \ldots, t_{N}$ be the zeros of $f_{1}$. Then, by the continuity, there exist neighborhoods $V_{t_{1}}, V_{t_{2}}, \ldots, V_{t_{N}}$ for all small $\varepsilon$, so that $f_{1}+\varepsilon f_{2}$ has exactly one zero in each $V_{t_{i}}$. On the other hand, $f_{1}+\varepsilon f_{2} \neq 0$ if $t \in T \backslash \bigcup_{i}\left(V_{t_{i}}\right)$ and $\varepsilon>0$ is sufficiently small. By using (2.5)-(2.7), Lemma 2.2, and the identity $\operatorname{sign}(a+b)=\operatorname{sign}\left(|a|^{p-1} \operatorname{sign} a+|b|^{p-1} \operatorname{sign} b\right)$, we have

$$
\begin{aligned}
\sigma(\varepsilon) & =S_{c}\left(f_{1}+\varepsilon f_{2}\right) \leq \tilde{Z}_{c}\left(f_{1}+\varepsilon f_{2}\right) \leq S_{c}\left(h_{1}+\varepsilon h_{2}\right) \\
& =S_{c}\left(Q_{p} h_{1}+Q_{p}\left(\varepsilon h_{2}\right)\right)=S_{c}\left(Q_{p} h_{1}+\varepsilon^{p-1}\left(Q_{p} h_{2}\right)\right) \\
& \leq S_{c}\left(\lambda_{1}^{-q} Q_{q} f_{1}+\varepsilon^{p-1} \lambda_{2}^{-q} Q_{q} f_{2}\right) \\
& =S_{c}\left(Q_{q} f_{1}+Q_{q}\left(\varepsilon^{(p-1) /(q-1)}\left(\lambda_{1} / \lambda_{2}\right)^{q /(q-1)} f_{2}\right)\right) \\
& =S_{c}\left(f_{1}+\varepsilon^{(p-1) /(q-1)}\left(\lambda_{1} / \lambda_{2}\right)^{q /(q-1)} f_{2}\right) \\
& =\sigma\left(\varepsilon^{(p-1) /(q-1)}\left(\lambda_{1} / \lambda_{2}\right)^{q /(q-1)}\right) .
\end{aligned}
$$

Iterating this inequality for $0<\varepsilon<1$, we obtain $\sigma(\varepsilon) \leq \sigma\left(\varepsilon_{0}\right)$, where $\varepsilon_{0}$ can be made arbitrarily close to zero (due to $1<q<p<\infty$ ), so that we may assume that $\sigma\left(\varepsilon_{0}\right)=N$. Consequently, $\sigma(\varepsilon) \leq N$ for $0<\varepsilon<1$. But then also $\sigma(1)=S_{c}\left(f_{1}+f_{2}\right) \leq N$ for otherwise one can choose $\varepsilon<1$ so close to 1 that $\sigma(\varepsilon)>N$.

Now, we turn to prove part (b). Taking $\lambda_{1}=\lambda_{2}, \varepsilon=1$ in (2.12), we get $S_{c}\left(f_{1}+f_{2}\right)=$ $\tilde{Z}_{c}\left(f_{1}+f_{2}\right)$. Lemma 2.4 is proved.

For a spectral function $f$, let $t_{1}<t_{2}<\cdots<t_{m}$ be all its zeros on $\mathbb{T}$, and let $s_{k}:=\left(t_{k}+\right.$ $\left.t_{k+1}\right) / 2, k=1, \ldots, m, t_{m+1}=t_{1}+2 \pi$ be the midpoints of the intervals between them.

Lemma 2.5. For $1<q \leq p<\infty$, a spectral function $f$ is odd with respect to each of its zeros $t_{k}$, that is, $f\left(t_{k}-t\right)=-f\left(t_{k}+t\right)$, and is even with respect to each $s_{k}$. Moreover, the number of zeros is even, $m=2 n$, and the points $t_{k}$ are equidistant on $\mathbb{T}$. The $f$ is periodic with period $2 \pi / n$.

Proof. Let $\left(f, \lambda^{q}\right) \in \Gamma(p, q, G)$. Then by [6], $\lambda=\|f\|_{q}$, and for each $k, f\left(t_{k} \pm t\right)$ is also a spectral function with the same $\lambda$. Therefore, $F(t)=f\left(t_{k}-t\right)+f\left(t_{k}+t\right)$ has a zero at $t=0$ without sign change. By (b) of Lemma 2.4, this function $F(t)$ must be zero.

The proof of Lemma 2.5 is complete. 
Lemma 2.6 (see [6]). Let $G$ be a B-kernel, $n \in \mathbb{N}, 1<p, q<\infty$. Then, $\Gamma_{2 n}(p, q, G) \neq \varnothing$. Moreover, if $\left(f, \lambda^{q}\right) \in \Gamma_{2 n}(p, q, G)$, then the function $f:=(G * h)+\beta$ satisfies the following conditions:

$$
f\left(x+\frac{\pi}{n}\right)=-f(x), \quad \forall x \in[0,2 \pi),
$$

with $\beta=0$, and the simple zeros of $f$ are equidistant on $T$, and

$$
h\left(t+\frac{\pi}{n}\right)=-h(t), \quad \forall t \in[0,2 \pi)
$$

Lemma 2.7. Let $G$ be a B-kernel. For $n \in \mathbb{N}, 1<q \leq p<\infty$, if $\left(f, \lambda^{q}\right) \in \Gamma_{2 n}(p, q, G)$. Then, there exists $h \in D_{n}$, such that $\lambda=\|f\|_{q}=\|G * h\|_{q}$.

Proof. For $\left(f, \lambda^{q}\right) \in \Gamma_{2 n}(p, q, G)$, by (2.7), and Lemma 2.6, we have $f=(G * h)(x)$. We choose $h\left(x_{0}\right) \geq 0, x_{0} \in[0, \pi / n)$, then $h\left(x_{0}\right) \sin n x_{0} \geq 0, x_{0} \in[0, \pi / n)$. For $x \in \mathbb{T}$, there exists a $i, i=1, \ldots, 2 n$, such that $x \in[(i-1) \pi / n, i \pi / n)$. Since $h(x+\pi / n)=-h(x)$. Thus

$$
h(x) \sin n x=h\left(x_{0}+\frac{(i-1) \pi}{n}\right) \sin \left(n\left(x_{0}+\frac{(i-1) \pi}{n}\right)\right)=h\left(x_{0}\right) \sin n x_{0} \geq 0 .
$$

Combining (2.14), we get $h \in D_{n}$, and $\lambda=\|f\|_{q}=\|G * h\|_{q}$. The proof of Lemma 2.7 is complete.

\section{Upper estimate of Bernstein $n$-width}

Following some ideas of Buslaev [4], Tikhomirov [1], Chen and Li [7], and Chen [5], the proofs of our main results are based on some iteration process which starts with an arbitrary function $h_{0} \in L^{p}$ with mean value zero and produces a sequence of functions $h_{k}$, and then a subsequence of their integrals $f_{k}$ converges to a spectral function $f$.

First, we take some $h_{0} \in L^{p}$ such that $\left\|h_{0}\right\|_{p}=1, h_{0} \perp 1$. Let

$$
f_{0}(x)=\left(G * h_{0}\right)(x)+\beta_{0}
$$

where $\beta_{0}$ satisfies the condition:

$$
\inf _{c \in \mathbb{R}}\left\|\left(G * h_{0}\right)+c\right\|_{q}=\left\|\left(G * h_{0}\right)+\beta_{0}\right\|_{q}, \quad 1<q<\infty .
$$

Next, we construct the sequences of functions $\left\{h_{k}\right\}$ and $\left\{f_{k}\right\}$ as follows:

$$
\begin{aligned}
f_{k}(x) & =\left(G * h_{k}\right)(x)+\beta_{k}, \quad k=1,2, \ldots, \\
\left(Q_{p} h_{k+1}\right)(y) & =\mu_{k+1}^{-q} \int_{\mathbb{T}} G(x-y)\left(Q_{q} f_{k}\right)(x) d x, \quad k=0,1,2, \ldots,
\end{aligned}
$$

where $\beta_{k}$ is uniquely determined by the condition

$$
\left\|f_{k+1}\right\|_{q}=\inf _{c \in \mathbb{R}}\left\|\left(G * h_{k+1}\right)+c\right\|_{q}=\left\|\left(G * h_{k+1}\right)+\beta_{k+1}\right\|_{q}, \quad 1<q<\infty,
$$

and $\mu_{k+1}>0$ is determined by the condition $\left\|h_{k+1}\right\|_{p}=1,1<p<\infty$. 
Lemma 3.1. Let $1<p, q<\infty$. Then

$$
\left\|f_{k}\right\|_{q} \leq \mu_{k+1} \leq\left\|f_{k+1}\right\|_{q}, \quad k=1,2, \ldots
$$

Proof. By the Hölder's inequality, (2.7), and $\left\|Q_{p} g\right\|_{p^{\prime}}=\|g\|_{p}^{p-1}$, we have

$$
1=\left\|h_{k+1}\right\|_{p}^{p-1} \cdot\left\|h_{k}\right\|_{p} \geq\left\langle Q_{p} h_{k+1}, h_{k}\right\rangle \geq \mu_{k+1}^{-q}\left\|f_{k}\right\|_{q}^{q},
$$

which proves the first inequality in (3.6). We now use this first inequality and similarly prove the second inequality:

$$
\begin{aligned}
1 & =\left\|h_{k+1}\right\|_{p}^{p}=\left\langle Q_{p} h_{k+1}, h_{k+1}\right\rangle=\mu_{k+1}^{-q}\left\langle G * Q_{q} f_{k}, h_{k+1}\right\rangle \\
& \leq \mu_{k+1}^{-q}\left\|f_{k+1}\right\|_{q} \cdot\left\|Q_{q} f_{k}\right\|_{q^{\prime}}=\mu_{k+1}^{-q}\left\|f_{k+1}\right\|_{q} \cdot\left\|f_{k}\right\|_{q}^{q-1} \leq \mu_{k+1}^{-1}\left\|f_{k+1}\right\|_{q} .
\end{aligned}
$$

The proof of Lemma 3.1 is complete.

It follows from Lemma 3.1 that the construction of the sequence $\left\{f_{k}\right\}_{k=1}^{\infty}$ is unambiguous. Moreover, it follows from (3.6) that $\left\{\mu_{k+1}\right\}_{k=1}^{\infty}$ is monotonic nondecreasing sequence and tends to some number $\mu$. It is clear that

$$
\mu:=\lim _{k \rightarrow \infty} \mu_{k}=\lim _{k \rightarrow \infty}\left\|f_{k}\right\|_{q}>0 .
$$

Lemma 3.2. For each starting function $h_{0} \neq 0, h_{0} \perp 1$, the sequence $\left\{h_{k}\right\}_{k=1}^{\infty}$ of (3.4) contains a subsequence $\left\{h_{k_{i}}\right\}_{i=1}^{\infty}$ for which $\left\{f_{k_{i}}(x)=\left(G * h_{k_{i}}\right)(x)+\beta_{k_{i}}\right\}_{i=1}^{\infty}$ converges uniformly to a spectral function $f$ (with a spectral value $\lambda=\mu$ ).

Proof. By using the weak compactness of the unit ball of the space $L^{p}, 1<p<\infty$, one can choose a subsequence $\left\{h_{k_{i}}\right\}_{i=1}^{\infty}$ converging weakly to some $h$ with $\|h\|_{p}=1$, with $\left\{f_{k_{i}}\right\}_{i=1}^{\infty}$ converging uniformly to $f:=(G * h)+\beta$. It follows from (3.4) that $\left\{Q_{p} h_{k_{i}+1}\right\}_{i=1}^{\infty}$ converges uniformly because the operator $Q_{p}, 1<p<\infty$, preserves uniform convergence. Consequently, $\left\{Q_{p^{\prime}} Q_{p} h_{k_{i}+1}=h_{k_{i}+1}\right\}_{i=1}^{\infty}$ converges uniformly to some $v$ with $\|v\|_{p}=1$, where $1 / p^{\prime}+1 / p=1$. Let $k \rightarrow \infty$ in (3.4) and with $\mu$ in (3.9). Then, we can obtain

$$
\left(Q_{p} v\right)(y)=\mu^{-q} \int_{\mathbb{T}} G(x-y)\left(Q_{q} f\right)(x) d x .
$$

Now, we turn to prove that $(f, \mu)$ is a spectral couple. Since in the following inequality, $Q_{p} h_{k_{i}+1} \rightarrow Q_{p} v$ uniformly and $h_{k_{i}} \rightarrow h$ weakly in $L^{p}$,

$$
\left\langle Q_{p} h_{k_{i}+1}, h_{k_{i}}\right\rangle=\mu_{k_{i}+1}^{-q}\left\langle Q_{q} f_{k_{i}}, h_{k_{i}}\right\rangle \geq \mu_{k_{i}+1}^{-q}\left\|f_{k_{i}}\right\|_{q}^{q} \longrightarrow \mu^{-q} \cdot \mu^{q}=1,
$$

which implies $\left\langle Q_{p} v, h\right\rangle \geq 1$. On the other hand, by the Hölder's inequality, and $\|v\|_{p}=\|h\|_{p}=1$, we get

$$
\left\langle Q_{p} v, h\right\rangle \leq\|v\|_{p}^{p-1} \cdot\|h\|_{p}=1
$$

Therefore, the case of equality can occur only if $\left|Q_{p} v\right|^{p^{\prime}}=|h|^{p}$, sign $Q_{p} v=\operatorname{sign} h$ almost every, or, equivalently, if $v=h$. Comparing (3.10) with (2.7), we get $\mu=\lambda$.

The proof of Lemma 3.2 is complete. 
For convenience, we denote by $\left(G, \lambda_{n}\right)$ all the function $h_{n}$, where $h_{n}$ is sufficiently

(i)

$$
\left\|G * h_{n}\right\|_{q}=\lambda_{n}:=\lambda(p, q, G)=\lambda_{n}\left\|h_{n}\right\|_{p}
$$

(ii)

$$
\int_{0}^{2 \pi} G(x-y)\left(Q_{q} G * h_{n}\right)(x) d x=\lambda_{n}^{q}\left(Q h_{n}\right)(y) d y, \quad y \in \mathbb{T} .
$$

In what follows, we need to convolute $G$ with periodic kernel for

$$
\phi_{\sigma}=\phi(\sigma, t):=\frac{1}{\sqrt{2 \pi}} \sum_{n=-\infty}^{\infty} \exp \left[-\frac{1}{2 \sigma^{2}}(t-2 n \pi)^{2}\right]
$$

$\sigma>0$. It is known that $[8]$

(i) $Z_{c}^{\star}\left(\phi_{\sigma} * f\right) \leq S_{c}(f)$,

(ii) $\lim _{\sigma \rightarrow 0^{+}} \phi_{\sigma^{*}} * f=f$ uniformly holds for every continuous function $f$ with $2 \pi$-period.

Let $G$ be a $B$-kernel. $G_{\sigma}:=\phi_{\sigma} * G$ is said to be the mollification of $G$ by $\phi_{\sigma}$. It is easily verified that $G_{\sigma}$ is a $B$-kernel.

Lemma 3.3 (see [5]). Suppose $h_{n, \sigma} \in\left(G_{\sigma}, \lambda_{n, \sigma}\right)$, where $\lambda_{n, \sigma}:=\lambda_{n}\left(p, q, G_{\sigma}\right)$. Then

(i) $\lim _{\sigma \rightarrow 0^{+}} \lambda_{n, \sigma}=\lambda_{n}$,

(ii) there exists a sequence of real number $\sigma_{k}>0$ such that $\sigma_{k} \rightarrow 0^{+}$and the corresponding sequence of continuous functions $\left\{h_{n, \sigma_{k}}\right\}_{k=1}^{\infty}$ is convergent uniformly on $\mathbb{T}$,

(iii) denote $h_{n}(x)=\lim _{k \rightarrow \infty} h_{n, \sigma_{k}}(x)$, then $h_{n} \in\left(G, \lambda_{n}\right)$.

We recall an equivalent definition on the Bernstein $n$-width of a linear operator $P$ from a linear normed space $X$ to $Y$.

Definition 3.4 (see [2, page 149]). Let $P \in L(X, Y)$. Then, the Bernstein $n$-width is defined by

$$
b_{n}(P(X), Y)=\sup _{X_{n+1}} \inf _{\substack{P x \in X_{n+1} \\ P x \neq 0}} \frac{\|P x\|_{Y}}{\|x\|_{X}}
$$

where $X_{n+1}$ is any subspace of $\operatorname{span}\{P x: x \in X\}$ of dimension $\geq n+1$.

Lemma 3.5. Let $G$ be a B-kernel. For each $p \in(1, \infty)$ and $n=1,2, \ldots$, then

$$
b_{2 n-1}\left(\tilde{B}_{p} ; L^{p}\right) \leq \lambda_{n}:=\lambda_{n}(p, p, G) .
$$


Proof. We first prove the theorem under the assumption that $G$ is sufficiently smooth, and $Z_{c}^{\star}(c+$ $G * h) \leq S_{c}(h)$ is true. An example of such function is $G_{\sigma}$, the mollification of $G$ by $\phi_{\sigma}$. Assume that $b_{2 n-1}\left(\widetilde{B}_{p} ; L^{p}\right)>\lambda_{n}$. From the definition of Bernstein $n$-width, there exists a $2 n$-dimensional linear subspace $L_{2 n}:=\operatorname{lin}\left\{g_{1}, g_{2}, \ldots, g_{2 n}\right\}$, and a number $\gamma>\lambda_{n}$, such that $L_{2 n} \cap \gamma S\left(L^{p}\right) \subseteq \widetilde{B}_{p}$, where $S\left(L^{p}\right)$ is the unit ball of $L^{p}$, that is,

$$
\min _{c+G * h \in L_{2 n}} \frac{\|c+(G * h)\|_{p}}{\|h\|_{p}}=\min _{f \in L_{2 n}} \frac{\|f\|_{p}}{\|h\|_{p}} \geq \gamma>\lambda_{n} .
$$

For every $f \in L_{2 n}, f=\sum_{j=1}^{2 n} \xi_{j} g_{j}$, define a mapping $f \rightarrow \xi=\left(\xi_{1}, \xi_{2}, \ldots, \xi_{2 n}\right) \in \mathbb{R}^{2 n}$. Using the similar method as that in [9, pages 214-216], we get $\|h\|_{p}=\left(\sum_{j=1}^{2 n} c_{j}\left|\xi_{j}\right|^{p}\right)^{1 / p}$, where $c_{j}=$ $\int_{(j-1) \pi / n}^{j \pi / n}|h(x)|^{p} d x, j=1, \ldots, 2 n$, and $c_{j}=\int_{0}^{\pi / n}|h(x)|^{p} d x=c_{1}, j=1, \ldots, 2 n$, if $h \in D_{n}$. By (3.18), we have

$$
\min _{\xi \in \mathbb{R}^{2 n} \backslash\{0\}} \frac{\left\|\sum_{j=1}^{2 n} \xi_{j} g_{j}\right\|_{p}}{\left(\sum_{j=1}^{2 n} c_{j}\left|\xi_{j}\right|^{p}\right)^{1 / p}}>\lambda_{n}
$$

Let

$$
S^{2 n-1}:=\left\{\xi: \xi=\left(\xi_{1}, \ldots, \xi_{2 n}\right) \in \mathbb{R}^{2 n}, \sum_{i=1}^{2 n} \xi_{i}=0, \sum_{i=1}^{2 n}\left|\xi_{i}\right|=2 \pi\right\} .
$$

For every vector $\xi \in S^{2 n-1}$, we take

$$
h_{0}^{\xi}(t)= \begin{cases}(2 \pi)^{-1 / p} \operatorname{sign} \xi_{k}, & \text { for } t \in\left(t_{k-1}, t_{k}\right), k=1, \ldots, 2 n, \\ 0, & \text { for } t=t_{k}, k=1, \ldots, 2 n-1,\end{cases}
$$

where $t_{0}=0, t_{k}=\sum_{i=1}^{k}\left|\xi_{i}\right|, k=1, \ldots, 2 n$, and let

$$
f_{0}^{\xi}(x)=\left(G * h_{0}^{\xi}\right)(x)+\beta_{0}, \quad 1<p<\infty,
$$

where $\beta_{0}$ satisfies the condition

$$
\inf _{c \in \mathbb{R}}\left\|\left(G * h_{0}\right)+c\right\|_{p}=\left\|\left(G * h_{0}\right)+\beta_{0}\right\|_{p}
$$

Next, for $p=q$, we consider the iterative procedure (3.3)-(3.4) beginning with $h_{0}^{\xi}$ and $f_{0}^{\xi}$ instead of $h_{0}$ and $f_{0}$, respectively. The analogues of Lemmas 3.1 and 3.2 hold. Moreover, for the limit element $f^{\xi}$, there exists $\widehat{\xi} \in S^{2 n-1}$ such that $f^{\widehat{\xi}}$ has at least $2 n$ simple zeros in $[0,2 \pi)$ (i.e., $S_{c}\left(f^{\widehat{\xi}}\right) \geq 2 n$ ). Indeed, let $O_{k}^{2 n-1}=\left\{\xi: \xi \in S^{2 n-1}, Z_{c}^{\star}\left(f_{k}^{\xi}\right) \leq 2 n-2\right\}$, where the function $f_{k}^{\xi}$ defined by (3.3). Clearly, the set $O_{k}^{2 n-1}$ is open in $S^{2 n-1}$. Let $H_{k}^{2 n-1}=S^{2 n-1} \backslash O_{k}^{2 n-1}$. Then, $H_{k}^{2 n-1}$ is a nonempty closed set, and that $H_{k+1}^{2 n-1} \subset H_{k}^{2 n-1}, k \in \mathbb{N}$. First, we prove that $H_{k}^{2 n-1}$ is nonempty. For fixed $0<x_{1}<x_{2}<\cdots<x_{2 n-1}<2 \pi$, let $\eta(\xi)=\left(\eta_{1}(\xi), \quad \eta_{2}(\xi), \ldots, \eta_{2 n}(\xi)\right)$, where

$$
\eta_{i}(\xi)= \begin{cases}\int_{\mathbb{T}} h_{0}^{\xi}(t) d t, & \text { for } i=1, \\ f_{k}^{\xi}\left(x_{i-1}\right), & \text { for } i=2, \ldots, 2 n .\end{cases}
$$


It is easily seen that $\eta(\xi)$ is a continuous and odd mapping. By Borsuk's theorem [10], there exists a $\bar{\xi} \in S^{2 n-1}$ such that $\eta(\bar{\xi})=0$. Then, $Z_{c}^{\star}\left(f_{k}^{\bar{\xi}}\right)=2 n-1$, that is, $\bar{\xi} \in H_{k}^{2 n-1}$. Thus, $H_{k}^{2 n-1}$ is a nonempty. Next, we prove $H_{k+1}^{2 n-1} \subset H_{k}^{2 n-1}, k \in \mathbb{N}$. Assume, on the contrary, there exists a $\tilde{\xi} \in H_{k+1}^{2 n-1}$, but $\tilde{\xi} \notin H_{k}^{2 n-1}$. Thus, $S_{c}\left(f_{k}^{\tilde{\xi}}\right) \leq Z_{c}^{\star}\left(f_{k}^{\tilde{\xi}}\right) \leq 2 n-2$ results in $S_{c}\left(Q_{q} f_{k}^{\tilde{\xi}}\right) \leq 2 n-2$. By (3.4), we get

$$
S_{c}\left(Q_{p} h_{k+1}^{\tilde{\xi}}\right) \leq 2 n-2, \quad S_{c}\left(h_{k}^{\tilde{\xi}}\right) \leq 2 n-2
$$

According to (3.3), we have $Z_{c}^{\star}\left(f_{k+1}^{\tilde{\xi}}\right) \leq 2 n-2$, namely, $\tilde{\xi} \notin H_{k+1}^{2 n-1}$. A contradiction follows from the above. We have constructed a system of nonempty closed nested sets. Their intersection is nonempty. Let $\widehat{\xi} \in \bigcap_{k=1}^{\infty}\left(H_{k}^{2 n-1}\right)$. According to Lemma 3.2, there exists $\left(f^{\widehat{\xi}}(x), \lambda^{p}\right) \in \Gamma(p, p, G)$ such that $\lim _{k \rightarrow \infty} f_{k}^{\hat{\xi}}(x)=f^{\widehat{\xi}}(x), x \in[0,2 \pi)$. Thus, $Z_{c}^{\star}\left(f^{\widehat{\xi}}\right) \geq 2 n-1$. In view of Lemma 2.3, zeros of $f^{\hat{\xi}}(x)$ are simple. Therefore, $S_{c}\left(f^{\widehat{\xi}}\right) \geq 2 n-1$. But since the function $f^{\hat{\xi}}(x)$ is periodic, we actually have $S_{c}\left(f^{\widehat{\xi}}\right) \geq 2 n$. We write $S_{c}\left(f^{\widehat{\xi}}\right)=2 N$.

For the spectral function $f^{\widehat{\xi}}$ corresponding to spectral value $\lambda(\widehat{\xi})$, by Lemma 2.7, and the nonincreasing property of Kolmogorov $n$-widths in $n$, and $d_{2 n}\left(\widetilde{B}_{p} ; L^{p}\right)=\lambda_{n}(p, p, G)$ [7], we have

$$
\lambda(\widehat{\xi}) \leq \lambda_{N}=d_{2 N}\left(\widetilde{B}_{p} ; L^{p}\right) \leq d_{2 n}\left(\widetilde{B}_{p} ; L^{p}\right)=\lambda_{n}
$$

Therefore, by Lemmas 3.1, 3.2, and (3.26), we have

$$
\min _{\xi \in \mathbb{R}^{2 n} \backslash\{0\}} \frac{\left\|\sum_{j=1}^{2 n} \xi_{j} g_{j}\right\|_{p}}{\left(\sum_{j=1}^{2 n} c_{j}\left|\xi_{j}\right|^{p}\right)^{1 / p}} \leq \frac{\left\|\sum_{j=1}^{2 n} \widehat{\xi}_{j} g_{j}\right\|_{p}}{\left(c_{1}\right)^{1 / p}\left(\sum_{j=1}^{2 n}\left|\widehat{\xi}_{j}\right|^{p}\right)^{1 / p}}=\left\|f^{\hat{\xi}}\right\|_{p}=\lambda(\widehat{\xi}) \leq \lambda_{n},
$$

which is contradicted with (3.19).

For a general $B$-kernel $G$, set $G_{\sigma}=\phi_{\sigma} * G$, and $h_{\sigma}=\phi_{\sigma} * h, \lambda_{n, \sigma}=\phi_{\sigma} * \lambda_{n}$. For $f=c+G * h \in$ $\widetilde{B}_{p}$, we set $f_{\sigma}=c+G_{\sigma} * h$. From the results obtained in the pervious case, we have

$$
\frac{\left\|G_{\sigma} * h+c\right\|_{p}}{\left\|h_{\sigma}\right\|_{p}}=\frac{\left\|f_{\sigma}\right\|_{p}}{\left\|h_{\sigma}\right\|_{p}} \leq \lambda_{n, \sigma}
$$

According to Lemma 3.3, we get $\|G * h+c\|_{p} /\|h\|_{p} \leq \lambda_{n}(p, p, G)$. Therefore, we obtain $b_{2 n-1}\left(\widetilde{B}_{p} ; L^{p}\right) \leq \lambda_{n}(p, p, G)$. The proof of Lemma 3.5 is complete.

\section{Proof of theorem}

Now, we consider the proof of Theorem 1.3.

Proof. By Lemma 3.5, if $G$ is $B$-kernel, for each $p \in(1, \infty)$ and $n=1,2, \ldots$, we have $b_{2 n-1}\left(\tilde{B}_{p} ; L^{p}\right) \leq \lambda_{n}(p, p, G)$. On the other hand, by [5], for each $1<p \leq q<\infty$ and $n=1,2, \ldots$, then $b_{2 n-1}\left(\widetilde{B}_{p} ; L^{q}\right) \geq \lambda_{n}(p, q, G)$. Thus, we have $b_{2 n-1}\left(\widetilde{B}_{p} ; L^{p}\right)=\lambda_{n}(p, p, G)$ for $p \in(1, \infty)$ and $n \in \mathbb{N}^{+}$. The result $(1.8)$ is obvious since $s_{2 n}\left(\widetilde{B}_{p} ; L^{p}\right)=\lambda_{n}(p, p, G)$ [5]. Theorem 1.3 is proved completely. 


\section{Acknowledgments}

The author would like to thank the referees for their valuable comments, remarks, and suggestions, which help to improve the content and presentation of this paper. Project supported by the Natural Science Foundation of China (Grant no. 10671019), Research Fund for the Doctoral Program Higher Education (no. 20050027007), and Scientific Research Fund of Zhejiang Provincial Education Department (no. 20070509).

\section{References}

[1] V. M. Tikhomirov, Nekotorye voprosy teorii priblizhenii, Izdatel'stvo Moskovskogo Universiteta, Moscow, Russia, 1976.

[2] A. Pinkus, $n$-Widths in Approximation Theory, vol. 7 of Ergebnisse der Mathematik und ihrer Grenzgebiete, Springer, Berlin, Germany, 1985.

[3] G. G. Magaril-Il'yaev, "Mean dimension, widths and optimal recovery of Sobolev classes of functions on line," Mathematics of the USSR-Sbornik, vol. 74, no. 2, pp. 381-403, 1993.

[4] A. P. Buslaev, G. G. Magaril-Il'yaev, and N. T'en Nam, "Exact values of Bernstein widths for Sobolev classes of periodic functions," Matematicheskie Zametki, vol. 58, no. 1, pp. 139-143, 1995 (Russian).

[5] D. R. Chen, "On the $n$-widths of some classes of periodic functions," Science in China. Series A, vol. 35, no. 1, pp. 42-54, 1992 (Chinese).

[6] G. S. Fang, "Eigenvalues of integral equations," Journal of Beijing Normal University. Natural Science, vol. 37, no. 6, pp. 720-725, 2001 (Chinese).

[7] H. L. Chen and C. Li, "Exact and asymptotic estimates for $n$-widths of some classes of periodic functions," Constructive Approximation, vol. 8, no. 3, pp. 289-307, 1992.

[8] S. Karlin, Total Positivity. Vol. I, Stanford University Press, Stanford, Calif, USA, 1968.

[9] S. Yongsheng and F. Gensun, Approximation Theory of Functions II, Beijing Normal University Press, Beijing, China, 1990.

[10] K. Borsuk, "Drei Sätze über die $n$-dimensional euklidische Sphäre," Fundamental Mathematics, vol. 20, pp. 171-191, 1933. 